\title{
4 Growing Pueblo data sovereignty
}

\author{
Michele Suina and Carnell T. Chosa
}

\section{Introduction}

Indigenous Data Sovereignty is a global phenomenon. Big data and the rapidly changing and increasingly powerful data technologies mean Indigenous Peoples world-wide experience many of the same data-related challenges. Yet Indigenous Data Sovereignty is, at its heart, a local phenomenon. A primary claim of Indigenous Data Sovereignty is of the data rights of the Indigenous data to whom the data pertain. Those Indigenous Peoples are most frequently not national populations but tribes, communities and individual Tribal Nations. This means that the advocacy work, data capacity building activity and enactment of Indigenous leadership in relation to Indigenous Data Sovereignty must also be at the local level. It is this local level enactment of Indigenous Data Sovereignty and Indigenous data governance that is the key focus of this chapter.

While relevant at all levels of Indigenous Data Sovereignty, at the local level, relationships and strategic partnerships are critical ingredients in the necessary mobilizing of Indigenous Nations and Peoples to transform the data landscape. It is relationships that provide the impetus and authority to shift the power back to Indigenous Nations to determine what data are necessary and what data are meaningful to inform Indigenous-led decision making in relation to policy. Efforts to advance Indigenous Data Sovereignty across all levels are growing in the United States. These include the drafting of the United States Indigenous Data Sovereignty Network, Indigenous Governance Principles at the University of California, Los Angeles "Policy Forum: The Governance of Indigenous Data" in May 2017. There is, however, still significant effort required to generate a greater awareness and understanding of the global Indigenous Data Sovereignty movement among tribes and tribal leadership. Before adopting Indigenous Data Sovereignty frameworks, tribal leaders need to be convinced of its potential to support local tribal efforts to control information and data about their people, lands, resources and all other aspects of their lives.

In this chapter, we will reflect on collaborative efforts between the Albuquerque Area Southwest Tribal Epidemiology Center (AASTEC) and the Santa Fe Indian School Leadership Institute (LI). This collaboration began in 2017 with the purpose of supporting local efforts to transform Indigenous health data as 


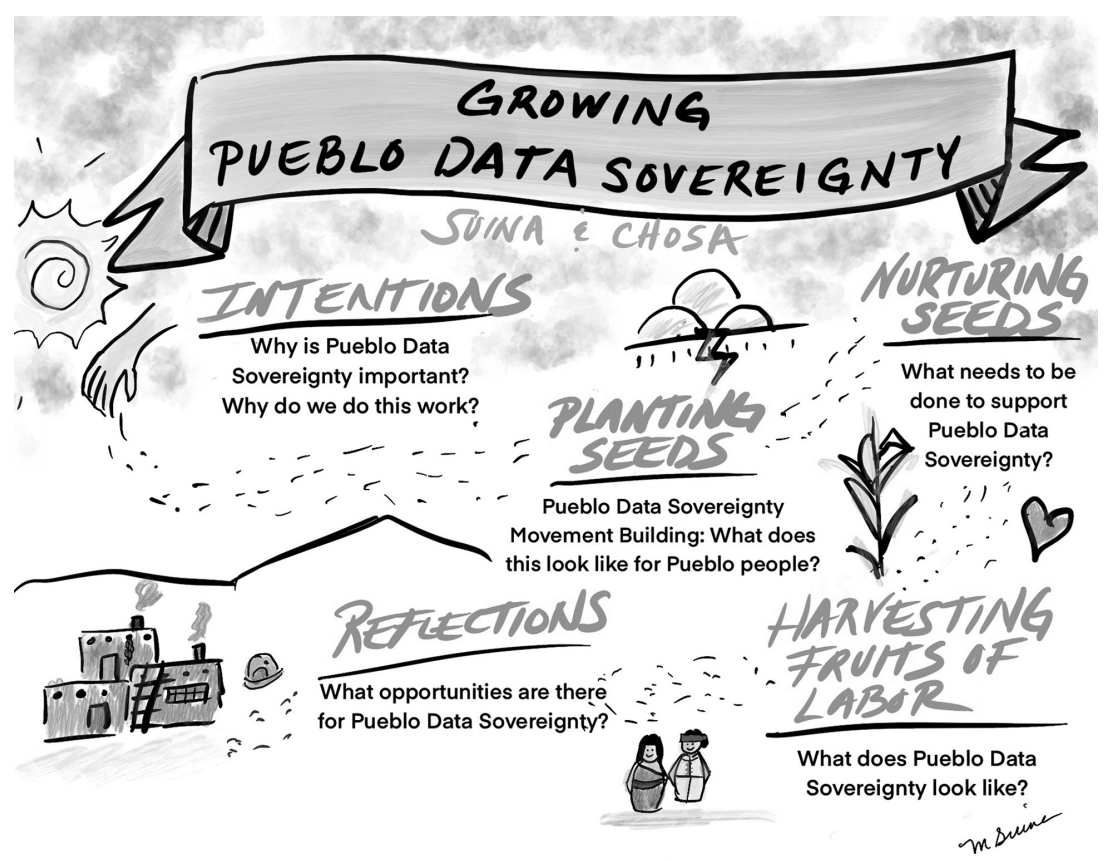

Figure 4.1 Growing Pueblo data sovereignty.

tribal-serving entities. We will also identify types of partnerships and community engagement necessary to move beyond awareness toward action and enactment of Indigenous Data Sovereignty. Finally, we will explore some lessons learned about how to grow Pueblo data sovereignty, to control data about Pueblo Peoples and Nations, by drawing from our positionality as Pueblo tribal members. These lessons have salience for other Indigenous Peoples in their Indigenous Data Sovereignty endeavors.

We have organized our chapter using a farming analogy, because farming and the values ${ }^{1}$ associated with caring for our Mother Earth, her plant and animal life, and humanity around us are central to the vitality of Pueblo People. Data can also play a central role for our People when we define for ourselves what data is most meaningful and what data can best contribute to the greater good of our People. We divide our chapter into five sections that each address a key Pueblo data sovereignty enactment question and the formation and flow of these are depicted in our Pueblo data sovereignty graphic (Figure 4.1) These five sections are:

1. intentions - Why is Pueblo data sovereignty important? Why do we do this work?

2. planting seeds - Pueblo data sovereignty movement building: what does this look like for Pueblo People? 
3. nurturing seeds - what needs to be done to support Pueblo data sovereignty?

4. harvesting fruits of labor-what does Pueblo data sovereignty look like?

5. reflections - what opportunities are there for Pueblo data sovereignty?

\section{Intentions-Why is Pueblo data sovereignty important? Why do we do this work?}

As Pueblo social justice practitioner-scholars, we were raised in our respective Pueblos (Cochiti and Jemez Pueblos, New Mexico, USA) connected to the Pueblo way of life $e^{2}$ unique to our individual Pueblos. This background has shaped the work we both do today to advocate for Pueblo data sovereignty. Contribution and service to the community are also important Pueblo core values that guide our efforts to support Pueblo efforts to control data about us and how we conceptualize the purpose of Pueblo data (Chosa, 2017). From this perspective, data should contribute to the well-being and be in the service of the Nations and Peoples it is about. Data must not exploit, stigmatize or create a disconnection from what is most important to Pueblo Peoples by invalidating our knowledge systems. But perhaps most importantly, data must contribute to the preservation of Pueblo governance, self-determination and knowledge that has been entrusted to us by generations that have come before us.

"Aboriginal occupancy of the Southwest by the Pueblo people is estimated at about ten thousand years before Christ" (Sando, 1992). The 20 Pueblo Nations located within the southwestern United States in New Mexico and Texas therefore have a longstanding presence that long predates the Spanish, Mexican and American occupation of Pueblo ancestral homelands that began in the 1500s. Although there are similarities among Pueblo Nations, each has its own way of life. Each also has its own unique governmental systems and languages that are foundational to their inherent sovereignty given by the Creator (Dozier Enos, 2015). For example, the 19 New Mexico Pueblo tribes share five different languages with their own dialects, including, Tewa, Towa, Tiwa, Keres, and Zuni. Both Zuni Pueblo (Zuni) and Jemez Pueblo (Towa) speak their own language.

Similar to Indigenous Peoples around the world, the Pueblo world was violently disrupted by settler colonialism. Our Pueblo forefathers and foremothers fought to protect our way of life during the Pueblo Revolt of 1680 that ejected the Spanish out of Pueblo territory for 12 years to end Spanish tyranny. Pueblo Peoples remained in our ancestral homelands and were not relocated which allowed us to maintain our connection to this day to where our ancestors also inhabited. Despite colonial efforts to assimilate Pueblo Peoples into Euro-American society to leave our own ways behind, Pueblos remain strongly connected to our ancestral ways of being. This continuation of our strong Pueblo identity often is attributed to the strong stance our Pueblo ancestors took to expel the Spanish colonizers in order to protect all that was important to our existence - our way of life.

Our Pueblo Peoples have preserved our cultural knowledge, languages and way of life through oral transmission from one generation to the next. Each Pueblo still maintains their rich traditional calendar and ceremonial activities throughout 
the year that reaffirm our core values and cultural knowledge that our ancestors fought to keep (conversation with Regis Pecos). Community-wide participation in our ceremonial life has been critical for passing on our cultural knowledge to younger generations. For example, during our traditional Pueblo corn dances it is common to see our elder tribal members participating alongside our youngest tribal members that just have barely started school. Holding onto knowledge vital to maintaining who we are as Pueblo Peoples and Nations is critical for upholding Pueblo sovereignty. Therefore, respecting, nurturing and carrying forward our knowledge and way of life has been ingrained in us as Pueblo Peoples. Maintaining our Pueblo knowledge is a heightened priority due to external colonial threats to dismantle who we are as Indigenous Peoples. Despite governmental policies to assimilate us into mainstream US society and erase us, our people still hold onto who we are as Pueblo Peoples even though cultural and language loss across Pueblos is a reality.

In recent times information and data about us, such as health and education statistics, generated by external interests, has become another area that we are entrusted to protect and ensure will benefit our people. Indigenous Data Sovereignty has become an important tool to enable us, in this data space to preserve, fight for and win, and treasure all that is important to us now and in the future. Data sovereignty, as a movement, brought together the respective organizations of the authors, the AASTEC ${ }^{3}$ and the Santa Fe Indian School Leadership Institute. ${ }^{4}$ In 2017 we joined forces to support local tribal efforts in the Albuquerque area to gain control over their data by convening a Community Institute. Best described as a native policy convener think tank, the purpose of this institute was to learn how tribal serving organizations can best support tribal Indigenous Data Sovereignty efforts.

Participants at the 2017 Community Institute recognized that data sovereignty is a process that would initially involve strategic partnerships. Within this, tribalserving organizations, such as AASTEC, would need to provide data-related support and technical assistance to tribal leaders and community members. The ultimate goal is tribes assuming ownership of their own data process including design, collection, analysis, reporting, storage and use of data. Establishment of tribal data policies defined by tribes and informed by local tribal data expertise were viewed as critical elements for tribes to achieve data sovereignty. Tribalserving organizations, such as AASTEC and the Leadership Institute were tasked with playing a supportive role, helping to cultivate technical expertise by providing data-related workshops and training opportunities at the tribal level, as well as an advocacy role to influence change among external data systems (i.e., government, university, etc.) that capture tribal data. While the Community Institute provided AASTEC and the Leadership Institute with a roadmap for how best to support data sovereignty among the 27 tribes, Pueblos, Bands and Nations in the Albuquerque area, it also provided the opportunity for focused work with the Pueblos led by the Leadership Institute and initiating deeper thinking about how to apply Pueblo teachings to data sovereignty. These elements are described in the following sections of the chapter. 


\section{Planting seeds - Pueblo data sovereignty movement building: what does this look like for Pueblo people?}

Looking at ways tribal communities already engage within is critical for understanding how to create a sustainable movement to achieve Pueblo data sovereignty. The work of co-author Chosa and the Leadership Institute of engaging Pueblo communities that draw from cultural-based philosophies provides rich examples of what movement building driven by Indigenous values looks like. The Leadership Institute creates and provides intentional opportunities where Native Peoples convene to develop solutions through program and policy recommendations from participants based on Pueblo cultural values.

Co-author Chosa (2016) has developed innovative community engagement opportunities for youth drawing from his own Pueblo's teachings and language to let young people know they belong to and are needed by their community. For example, in 2005 the Leadership Institute created an internship program to reconnect Pueblo youth with their tribal communities. The internship program, now called New Mexico Summer Youth Tribal Employment, ensured host sites provided opportunities for meaningful engagement between youth and their community to occur, making sure that internship placements aligned with both the students' college major and community-expressed needs. As a result, participating communities have retained their youth and the vital contributions youth make to their communities, strengthening the interrelationship between community and youth. This approach was inspired by Chosa's upbringing in Jemez Pueblo. This upbringing modeled participating and contributing to community as coming from having an unwavering sense of belonging to and love for community that is reciprocated by the community. Chosa refers to this interaction in his Towa language as "Attaching Your Heart" meaning when one makes themselves present to support or contribute to a family or community event. Oftentimes, this concept of Attaching Your Heart is also used as an invitation or a call to action to participate or engage in a larger initiative. Additionally, Chosa shares how the concept of belonging is critically tied to or is in relationship with the one who is attaching their heart. When one feels a sense of belonging, they are more likely to attach their heart, and vice versa; by attaching your heart, your level of belonging is strengthened.

Applying what Chosa terms as "homegrown models" based on local Pueblo philosophies and values such as Attaching Your Heart and belonging provides important frameworks for data sovereignty. First, they allow Pueblo Peoples to see themselves reflected back to themselves, leading to an increase in their engagement. The framework can also be reapplied to the very issues that necessitate engagement of Pueblo people. For example, because most Pueblo data is generated by outside entities or initiators with different values and ideas of who we are, the data does not really belong to us. We are not able to attach our heart to the data because it is not us nor does it reflect who we really are. Therefore, data about Pueblo Peoples may be viewed as irrelevant, invalid, unreliable and at worst be seen as harmful because it typically describes us in deficit terms (Walter 
\& Suina, 2018). Creating our own data for our own purposes and based on our own values, changes the way that data is viewed; they truly belong to us. Data that belongs to us would help us to attach our hearts and use the data to create meaningful programs and opportunities for our Peoples. Examining who initiates engagement and generates data is also critical for understanding where opportunities exist to encourage Pueblo participation in data creation and use.

For Pueblos, interdependence on one another has been critical for ensuring our existence. The interrelationship between the community and the individual plays a major role in maintaining interdependence to benefit the greater good. Similarly, interdependence among external tribal-serving entities is necessary to maximize our service to tribes and to provide relevant programming and resources to benefit the Peoples we serve. Partnering with the Leadership Institute in 2017 was an intentional strategy for AASTEC to elevate Indigenous Data Sovereignty among the tribes in our area. Based at the Santa Fe Indian School, a tribally controlled and operated school, the Leadership Institute is an important entity as it has served as a catalyst since 1997 to create discourse among tribes. The other central role of the Leadership Institute is to train community members and specifically youth on public policy issues in order to create systemic change starting within tribal communities. Because of their long track record utilizing Indigenous centered values and philosophies to engage people, the Leadership Institute is influential among tribal leaders, policy makers and tribal grassroots organizers.

\section{Nurturing seeds-What needs to be done to support Pueblo data sovereignty?}

In July 2018, the Leadership Institute convened the Pueblo Convocation ${ }^{5}$ on Education that brought together nearly 700 Pueblo people to reimagine what education should look like for Pueblo students. This event was in response to the landmark court decision that found that the state of New Mexico violated the constitutional rights of Native American students to a sufficient education in the Yazzie/Martinez lawsuit. ${ }^{6}$ During the Convocation, Pueblo Peoples put their hearts and minds together to begin to develop plans for a desired educational system for their children and youth to prepare for the 2019 state legislative session. Data sovereignty was included among the 13 breakout sessions. The outcome of these sessions was a set of recommendations that were presented to the 20 Pueblo governors for inclusion in a tribal resolution to guide efforts for reforming an inadequate education system.

The Convocation breakout session entitled "Data Sovereignty, Effective Uses of Data" discussed how data could be more effectively used by the Pueblos to make stronger arguments for change across the educational spectrum. Participants were asked during the breakout session what policies are needed so that tribes can control and influence data about them and for the data that does exist to be more effectively used. A determination of this discussion was that policies are needed that establish clear guidelines for Pueblo data. These include guidelines that identify: what types of data can be collected; how data should be collected; appropriate 
data sources; why data is being collected; and how data can be better used to benefit Pueblo Peoples. It was also determined that clear guidelines were needed to establish who can access data and who owns and controls Pueblo-related data. Although there was recognition that each Pueblo would need to establish their own review boards and advisory boards to determine their own research agendas/priorities, data policies and guidelines, there was also the idea expressed that Pueblos could band together and form an office of research if they desired to do so.

Participants in the data sovereignty breakout session also expressed the need for training and education on data for those in leadership positions. This training would equip Pueblo leaders to advocate for data sovereignty and how to use data and support the community at large to understand the importance of data and to gain a basic understanding of data terminology. Regarding practical matters, funding was recognized as a significant need. The following Pueblo data sovereignty protocol recommendations were made as a result of the session's rich dialog that occurred. These protocols were included in the Pueblo resolution:

1. Pueblos must recognize the important role of data collection. Data collection is now an important part of governance and ownership and application to assist and support communities.

2. Pueblos must develop strategies on how we can use data to advance our initiatives at multiple levels.

3. Pueblos must actively develop data sharing agreements.

4. Pueblos must consider developing internal and external policies so Pueblos can control and influence data about them.

5. Advocate to redefine "evidence-based" research and data collection in ways that enable Pueblos to define what evidence looks like and enables locally conducted research, enable data collection to be used as evidence to support initiatives, and enable programs that Pueblos know work for them.

6. Pueblo leadership must support and advocate for increasing base budgets for the existing tribal serving centers and institutes to continue these groundbreaking initiatives to expand their work and extend technical support to the Pueblo governments and Pueblo programs.

The actions that grew from the Pueblo Convocation on Education offered direction for continued Pueblo data sovereignty efforts. They also remind us that achieving data sovereignty will rely on multiple and ongoing endeavors involving many individuals (i.e., Pueblo leadership, community members at large, technical assistance providers, etc.), but the initiators of Indigenous Data Sovereignty must be our own Pueblo People.

\section{Harvesting fruits of labor-What does Pueblo data sovereignty look like?}

Understanding what sovereignty means to Pueblo Peoples, instead of external definitions, is critical for informing Pueblo data sovereignty efforts and in creating 
data governance mechanisms that align with traditional Pueblo governance that protect our way of life. Santa Clara Pueblo scholar, Anya Dozier Enos' (2015) education research outlines what inherent sovereignty means to Pueblo people. Drawing from interviews conducted with Pueblo people, Dozier Enos describes Pueblo inherent sovereignty as being the core of life or the way of life of Pueblo people, using the term deep sovereignty to describe this concept. Dozier Enos further explains that deep sovereignty is fundamentally different from political sovereignty because it is not restricted to the Western legal definitions that limit actual sovereignty of American Indian tribes in the United States. Pueblo data sovereignty plays an important role in maintaining inherent Pueblo sovereignty because the enactment of data sovereignty is through governance and decision making related to data matters about Pueblo people and nations.

Pueblos remain vigilant in the protection of our way of life. From the Spanish colonial period to save their native religion from the brutal Spaniards, our Peoples have kept Pueblo knowledge close at hand and away from the non-Pueblo world to protect our sacred way of life (Suina, 1992). Cochiti Pueblo scholar and tribal leader, Joseph H. Suina (co-author M. Suina's father) reflected on what this strategy looks like for many Pueblos in more recent times:

Highly visible signs forbidding picture-taking, sketching and other forms of recording are posted on the outskirts as well as in the village center. Visitors' recording devices, such as cameras, can be impounded by tribal officials and violators are faced with a possible fine. Usually, the film is confiscated and a warning issued to the embarrassed offender. Occasionally a Pueblo village will be closed to the outside world for periods of two or three days to celebrate a private religious event. Not even the U.S. mail gets through. (p. 60)

Pueblos already have built-in mechanisms to protect their information from the outside world. Pueblos also have internal practices in place among their own people that limit access to certain types of information if a person is not yet ready for that information or if that information is not intended for that person (Suina \& Smolkin, 1994). Examining already existing systems and practices is essential for developing Pueblo data governance mechanisms that better align with Pueblo knowledge practices that are connected to our own epistemological, ${ }^{7}$ ontologi$\mathrm{cal}^{8}$ and axiological ${ }^{9}$ frameworks. While there is value in current data governance mechanisms such as research codes, data sharing agreements, research review boards and other methods for tribes to control their data, there must also be a connection to our Pueblo value systems and knowledge worlds to mirror our own processes. Otherwise, we run the risk of invalidating our own knowledge by not applying it to protect our information and data.

Pueblo data sovereignty has become a more recent example of how Pueblos are challenging outside impositions of what is valid, important and truly represented by data and asserting their rights to determine what data is necessary for their own purposes. During the 2018 Pueblo Convocation, several rich examples of Pueblo Nations taking on a collection of data for their own purposes emerged. 
For example, Pueblos are engaged in their own data collection for comprehensive community and land use planning efforts, understanding heritage language trends, and ensuring oversight of research by establishing research review mechanisms such as the establishment of an Institutional Review Board by one Pueblo. Pueblos are exercising their inherent rights as sovereign Nations to govern their data and information, as well as to create their own data.

\section{Reflections-What opportunities are there for Pueblo data sovereignty?}

In 2019, the Keres Children's Learning Center ${ }^{10}$ held their Fourth Annual Language Symposium showcasing how Montessori education is being used to revitalize heritage languages. Tribal leader and keynote speaker Joseph H. Suina reflected upon Pueblo Peoples' ability to adapt outside ways to fit them by stating, "The genius of Pueblo people, we take something and make it fit us". Suina further explained that rather than Pueblo Peoples changing to fit the outside way or idea, Pueblo Peoples incorporate what is useful while still remaining who we are without losing what is important to the Pueblo world at the expense of what is being introduced such as the adoption of different teaching methods. For example, traditional holistic Pueblo pedagogy and the Montessori Method both focus on educating the whole child and have been woven together to support language immersion to revitalize language in one Pueblo. Likewise, Pueblo data could better serve Pueblo Peoples by being in alignment with our own values and realities instead of those that are important to government agencies, funders, universities and others that have staked a claim on information about us. Data can then become a better fit without Pueblos having to give up what is most important to them.

As Pueblo advocates for Pueblo data sovereignty, Suina's words resonate. The question becomes, how can the Indigenous Data Sovereignty movement fit the Pueblos so we can make it our own? Having a better understanding of this essential element of Pueblo data sovereignty is critical for supporting a movement for lasting change that aligns with what is most important for Pueblo Peoples. The work of Laguna Pueblo scholar and tribal leader Richard Luarkie (2017) examines the relationship between Pueblo Peoples and data and reminds us that "data and analytics are part of our culture and way of life and are not new to Pueblo people". Luarkie explains that Pueblo Peoples have in the past and to this day rely on the use of oral-based and visual data sets that have been guided by what Luarkie terms a "holistic world data-view" derived from our own Pueblo values and principles. Data collection, analysis, reporting and usage are something our people have already been doing prior to colonization. Being able to identify commonalities between Western-based data and existing Pueblo data and cultural practices that are built into our way of life is a way to make a connection with Pueblo Peoples regarding the relevance of data to who we are rather than data being disconnected from us. For example, epidemiological health data provides us with information that marks significant life events such as when a person is 
born and when a person leaves this world; data is collected that acknowledges these important events. In the Pueblo world these important events are recognized by the entire community. Quantitative data and Pueblo cultural practices both recognize the importance of these life events that must be noted. We need to identify where other areas align and examine ways to share information in a way that respects cultural values and teachings. This is an ongoing role of the tribal serving entities such as Albuquerque Area Southwest Tribal Epidemiology Center and the Santa Fe Indian School Leadership Institute.

\section{Conclusion}

Growing Pueblo data sovereignty will involve further exploration to understand how Pueblo Peoples view data through our own cultural lens and teachings to guide what data looks like and should look like for our people. Nurturing Pueblo data will take multiple engaged Pueblo community partnerships that recognize the role communities must play in order to be the bearers and ultimately the recipients of the data harvest. Engaged partners external to our communities can contribute to Pueblo data sovereignty by helping to cultivate technical data skills among Pueblo Peoples and to advocate for change among external data systems that collect our data. Sustainable production of Pueblo data by Pueblo Peoples will be a significant contribution that we can pass onto future generations. By doing this, we will protect all that our ancestors fought for throughout our history to ensure we are connected to our Pueblo way of life to maintain our inherent sovereignty gifted to us by the Creator.

\section{Notes}

1 Pueblo core values are love, respect, compassion, faith, understanding, spirituality and balance.

2 Pueblo way of life encompasses a connection to where our ancestors lived and where we still live today, the practice of Pueblo spirituality and ceremonies, use of heritage languages, maintenance of traditional governance systems and other ways of existence that connect today's Pueblo Peoples to our ancestors.

3 Albuquerque Area Southwest Tribal Epidemiology Center in Albuquerque, New Mexico, USA, was established in 2006 and is one of 12 Tribal Epidemiology Centers serving American Indians and Alaska Natives throughout the USA and serves tribal communities in New Mexico, southern Colorado and west Texas to provide high-quality health data, culturally congruent epidemiology/surveillance, capacity development, program evaluation and health promotion/disease prevention services.

4 The Leadership Institute (LI), based at the Santa Fe Indian School in Santa Fe, New Mexico, USA, was established in 1997 to create a space for discourse on a wide range of public policy and tribal community issues challenging the vitality and spirit of the 22 Tribal Nations in New Mexico.

5 The Pueblo Convocation is another Leadership Institute programmatic component that brings together a larger number of participants (between 500 to 800 ) to engage in an in-depth exploration of an issue area that may need formal multi tribal support.

6 The consolidated lawsuit, Yazzie/Martinez v. State of New Mexico challenged the state's failure to provide students - especially low-income, Native American, English 
language learner (ELL), and students with disabilities - the programs and services necessary for them to learn and thrive, and challenged the state's failure to sufficiently fund these programs and services. On July 20, 2018, Judge Sarah Singleton ruled that all New Mexico students have a right to be college and career ready and that the state is failing to meet this obligation (http://nmpovertylaw.org/wp-content/uploads/2018/09 /Graphic-Yazzie-Martinez-Decision.pdf).

7 Epistemology refers to the part of philosophy that deals with knowledge. (Oxford online dictionary)

8 Ontology refers to philosophy that deals with the nature of existence. (Oxford online dictionary)

9 Axiology refers to the study of the nature, types, and criteria of values and of value judgments, especially in ethics (Merriam-Webster online dictionary).

10 Keres Children's Learning Center KCLC is a Montessori early childhood education center that immerses Cochiti Pueblo children in their Keres language. The mission of KCLC is to reclaim our children's education and honor our heritage by using a comprehensive cultural and academic curriculum to assist families in nurturing Keresspeaking, holistically healthy, community minded and academically strong students (https://kclcmontessori.org/).

\section{References}

Chosa, C.T. (2016). New engagement programming with youth. Journal of American Indian Education, 55(3), 12-29.

Chosa, C.T. (2017). Attaching your heart: pueblo community engagement. In Indigenous Innovations in Higher Education (pp. 165-180). Brill Sense.

Enos, A.D. (2015). Deep sovereignty: a foundation for indigenous sustainability. In Indigenous Innovation (pp. 25-42). Brill Sense.

Luarkie, R. (2017). Rethinking data through pueblo interpretations. In Indigenous Innovations in Higher Education (pp. 123-141). Brill Sense.

Sando, J.S. (1992). Pueblo Nations: Eight Centuries of Pueblo Indian History. Clear Light Pub.

Suina, J.H. (1992). Pueblo secrecy: result of intrusions. New Mexico Magazine, 70(1), $60-63$.

Suina, J.H., \& Smolkin, L.B. (1994). From natal culture to school culture to dominant society culture. In Cross-Cultural Roots of Minority Child Development (pp. 115-130). Lawrence Erlbaum Associates, Inc.

Walter, M., \& Suina, M. (2018). Indigenous data, indigenous methodologies and indigenous data sovereignty. International Journal of Social Research Methodology, 22, 1-11. 